Part of Journal of Research of the National Bureau of Standards, Volume 14, March 1935

\title{
A PRECISION CATHETOMETER
}

\author{
By Charles Moon
}

\section{ABSTRACT}

A cathetometer which was built for the special purpose of measuring the length of single-layer inductance coils is described. The instrument is suitable for precise measurements of length by comparison with a standard scale. Two microscopes with micrometer eyepieces are carried on a vertical shaft which is supported by a self-aligning type of ball bearing at the bottom and a V-block type of bearing at the top. These bearings allow the shaft to turn easily on its axis with no tendency to bend the shaft and thus vary the distance between the microscopes. A brief discussion is given of the error caused by inaccuratefocussing which was especially troublesome in measuring the length of inductances.

A cathetometer suitable for precise measurements has been built for the special purpose of measuring the length of inductance coils which are in the form of single-layer solenoids. It is a wall-type instrument intended only for measuring an unknown length by comparison with a calibrated line standard. A brief description is given because it may be useful to those who may have similar measurements to make.

The instrument is shown in figure 1 as used in measuring the length of a helix of wire. The two micrometer microscopes are carried on a steel shaft 1.5 inch in diameter. The shaft is supported by a lower bearing which rests on the table and an upper bearing which is screwed to the cabinet holding the inductance coil. The weight of the vertical column and the two microscopes. is carried by the lower bearing which is a double-row ball bearing of the self-aligning type. The outer race of this bearing is set in a brass collar which has a loose radial fit in the base plate, so that. a small horizontal adjustment can be made in any direction by means of four screws. The upper bearing consists of a hardened ball at the end of the shaft which is held lightly in a hardened Vblock by a thin bronze spring.

The vertical column rotates very easily and smoothly and may be accurately stopped at two positions by holding the arm A against either the tangent screw $S_{1}$ or $S_{2}$ by means of the thin spring $B$ at the end of the arm. $S_{1}$ is set for readings on the helix and $S_{2}$ for readings on the line standard.

The excellence of the instrument is attributed to the bearings and the stopping device which allow an easy shift in position with no. tendency to flex or stress the column in any way, and thus change the relative position of the two microscopes. 
Each microscope is mounted on a sliding carriage $\mathrm{C}$ with means for leveling. The carriage $\mathrm{C}$ is connected to a second carriage $\mathrm{D}$ by a screw which gives a small vertical adjustment. Both $\mathrm{C}$ and $\mathrm{D}$ can be securely clamped to the vertical column.

The microscopes are fitted with vertical illuminators and light sources which move with the telescopes. A 6-volt frosted bulb



Figure 1.-Precision cathetometer as mounted to measure the length of a singlelayer solenoid.

with a condensing lens and a green gelatine filter is placed in a short tube fixed at right angles to the microscope tube. The light falls on a thin glass plate tilted at an angle of $45^{\circ}$ so that it is reflected out through the objective to the ruled surface or wire. This type of illuminator, which allows the entire objective to be used, is greatly superior for these measurements to the type using a $45^{\circ}$ prism which covers approximately one-half of the objective. 
The adjustments of the cathetometer and sources of error are similar to those of any cathetometer of the comparator type. The source of error which is most difficult to eliminate in measuring the length of an inductance coil is that caused by faulty focussing of the microscopes.

With an aplanatic objective all rays from a given point will be brought to focus at a second point conjugate with the first. If the plane of the crosshairs is exactly at the focus a correct reading of the position of the object will be obtained whether the whole or a part of the objective is used. If the plane of the crosshairs is within the focus, the image formed by a pencil of rays through the upper margin of the objective will be displaced upward, and the image formed by a pencil through the lower margin will be displaced downward. If the plane of the crosshair is beyond the focus, the displacement in each case will be in the opposite sense. ${ }^{1}$ In practice, the displacements may be increased by chromatic abberation, ${ }^{2}$ spherical aberration, and coma, ${ }^{3}$ in the lens used. With an apparently good focus, the displacements of as much as four microns have been observed using a 4-inch objective.

While the difficulties from focussing are well known to a few, there is little discussion in the literature and unfortunately there seems to be no accepted method of determining when the focus is correct.

In this work, the most useful method of focussing was that of Cornu and Benoit ${ }^{4}$ in which the focus is adjusted by trial until the same reading of the micrometer is obtained when the light is caused to pass through opposite margins of the objective by means of a movable diaphragm. The improvement when using this method is mostly in the focus on the wires. On the line standard, where the accuracy of focussing can be judged roughly by the constancy of the calibration of the filar micrometer, the results of a series of calibrations after focussing by the Cornu method are not much more uniform than the results of another series obtained when the focussing was done by simply adjusting for best definition.

Another useful method of focussing is to adjust the microscope until no parallax results when the light source is moved. ${ }^{5}$ Optically, it is similar to the method of Cornu.

The instrument, equipped with microscopes having objectives of 10-centimeter working distance, has been used in making measurements on two different solenoids. When the column has been turned and brought back to its original position, the difference in the reading of each microscope has always been less than the error of observation. This indicates that the distance between the microscopes and the elevation of the instrument as a whole are maintained with a high order of precision.

\section{Washington, September 24, 1934.}

\footnotetext{
${ }_{1}$ Cornu and Benoit. Travaux et Mémoires du Bureau International des Poids et Mesures 10, 12 (1894). See also J. Guild. Proc. Phys. Soc. (London), 28, 242 (1915-16); Natl. Phys. Lab. Collected Researches Repts., 13, 229 (1916).

'J. Guild. Chromatic parallax. Natl. Phys. Lab. Collected Researches Repts., 14, 249 (1920); also Proc. Phys. Soc. (London), 29, 311 (1916-17).

3 Benoit and Guillaume. Travaux et Mémoires du Bureau International des Poids et Mesures, 10, 35 (1902).

4 See footnote 1 .

5 Wolff, Shoemaker, and Briggs. Bul. BS 12, 432 (1915) S256.
} 